\title{
Geometry deviation effects of railway catenaries on pantograph- catenary interaction: a case study in Norwegian Railway System
}

\author{
Yang Song $^{1} \cdot$ Tengjiao Jiang $^{1}$ (D) Petter Nåvik $^{1} \cdot$ Anders Rønnquist $^{1}$
}

Received: 30 April 2021 / Revised: 16 July 2021 / Accepted: 22 July 2021 / Published online: 12 August 2021

(C) The Author(s) 2021

\begin{abstract}
This paper presents a non-contact measurement of the realistic catenary geometry deviation in the Norwegian railway network through a laser rangefinder. The random geometry deviation is included in the catenary model to investigate its effect on the pantograph-catenary interaction. The dispersion of the longitudinal deviation is assumed to follow a Gaussian distribution. A power spectrum density represents the vertical deviation in the contact wire. Based on the Monte Carlo method, several geometry deviation samples are generated and included in the catenary model. A lumped mass pantograph with flexible collectors is employed to reproduce the high-frequency behaviours. The stochastic analysis results indicate that the catenary geometry deviation causes a significant dispersion of the pantograph-catenary interaction response. The contact force standard deviations measured by the inspection vehicle are within the scope of the simulation results. A critical cut-off frequency that covers $1 / 16$ of the dropper interval is suggested to fully describe the effect of the catenary geometry deviation on the contact force. The statistical minimum contact force is recommended to be modified according to the tolerant contact loss rate at high frequency. An unpleasant interaction performance of the
\end{abstract}

Yang Song

y.song_ac@hotmail.com

Tengjiao Jiang

Tengjiao.jiang@ntnu.no

Petter Nåvik

petter.r.navik@ntnu.no

Anders Rønnquist

anders.ronnquist@ntnu.no

1 Department of Structural Engineering, Norwegian University of Science and Technology, 7491 Trondheim, Norway pantograph-catenary can be expected at the catenary top speed when the random catenary geometry deviation is included.

Keywords Railway · Pantograph · Catenary · Geometry deviation $\cdot$ Non-contact measurement $\cdot$ Contact force

\section{Introduction}

In electrified railway systems, the catenary constructed along the railroad is used to power the electric train. The electric current is transmitted to the train through a sliding contact with a pantograph mounted on the vehicle roof, as illustrated in Fig. 1. The catenary's contact wire serves mechanically as the electrical path for the pantograph and the electrical current. The current collection quality is dominated by the mechanical interaction performance between the contact wire and the pantograph collectors.

Usually, the contact wire is hanged by several droppers to keep it as flat as possible or to have a certain amount of pre-sag, which has been proven beneficial to keep a stable contact with pantographs [1]. However, the installation error, temperature variance, insufficient maintenance and multiple impacts in long-term operation may result in the deviation of the catenary geometry with respect to its design position [2,3], which affects the sliding contact of the pantograph-catenary. As shown in Fig. 2, the deviation happens in longitudinal and vertical directions, which have been proven by the measurement data in our previous work [4]. Similar to the track irregularity [5], the catenary geometry deviation also has a stochastic nature, resulting in 


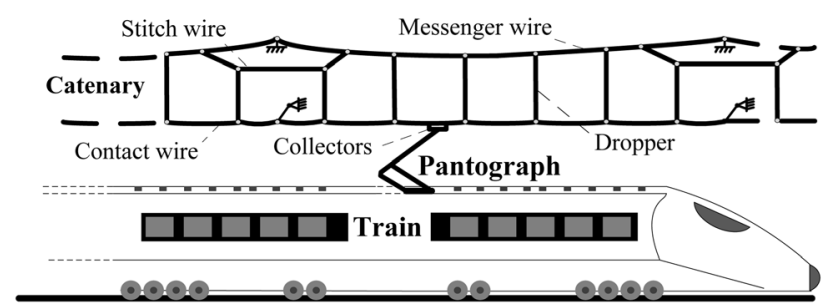

Fig. 1 Schematics of pantograph-catenary system

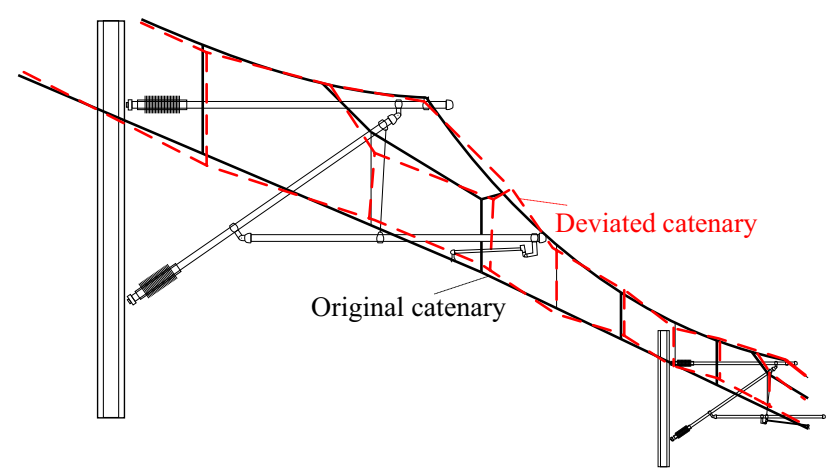

Fig. 2 Illustration of geometry deviation

an indeterministic response of pantograph-catenary interaction. Therefore, the realistic geometry deviation is desired to be measured and properly included in the numerical tools to evaluate the dispersion of pantographcatenary behaviour, which is the main topic of this paper.

As the most vulnerable part of the traction power system, the pantograph-catenary system has been a common research objective that attracts ever-increasing attention of scholars from the scientific community and the industry [6]. Due to the high cost and limited access of field tests, numerical modelling has been a mainstream approach to study the pantograph-catenary dynamics and has experienced an advanced development in the last several decades [7]. In the early stage of research, the catenary is normally assumed to be a mass-spring system [8], in which only the stiffness and mass distributions are considered without the contribution of wave propagation. With the increase of train speed, the catenary nonlinearity significantly affects the response [9]. That is why the finite element method has been the most popular approach to model the catenary. To achieve convincing numerical results, the measurement data from field tests are utilised to modify and validate numerical models, which has prompted new measurement and identification techniques [10,11]. Another state of the art is to digitalise the external disturbance from vehicletrack [12, 13], wind load [14] and other experimental factors and adequately include them in the numerical model. In order to improve the numerical efficiency, some scholars have devoted their attention to developing fast simulation techniques, such as the moving mesh [15], moving window [16] and offline integration method [17]. Commonly, the numerical models are used in the design phase to check the acceptance of the design strategy. Recently, the modelling of degradation has been an emerging technique to utilise the numerical model to predict service performance [18]. Based on this idea, the defective droppers [19, 20], irregularity [21], contact wire wear [22] and the tension variation [23] are correctly modelled and included in the assessment of pantographcatenary interaction. It is worthwhile to mention the works of Van et al. [24] and Gregori et al. [25]. The geometry deviation is considered in these two works when modelling the catenary, and the results indicate that the geometry deviation leads to a particular dispersion of the response. The geometry data from measurement can further support and validate the main conclusion of these two works.

From the above literature review, it is seen that most numerical simulations of pantograph-catenary interaction are performed based on design data without any geometry imperfections. Some works that attempt to include a random geometry deviation in the catenary model lack the support of measured geometry data. This paper presents a filed measurement of the catenary geometry based on a laser rangefinder, which can provide realistic contact wire geometry for the numerical simulation. Based on the Monte Carlo method, many contact wire geometry samples are generated, with the same distribution and frequency characteristics as the measurement data. The random geometry deviation is included in a catenary model built by the finite element method (FEM). In combination with a pantograph model with flexible collectors, the dispersion of response caused by the random geometry deviation is investigated with different cut-off frequencies and operating speeds.

The rest of this paper is organised as follows: Sect. 2 describes the measurement of catenary geometry. The catenary model with geometry deviation is built in Sect. 3 . The stochastic analysis of the contact force is performed in Sect. 4, and Sect. 5 states the conclusions.

\section{Measurement of catenary geometry}

To provide a realistic catenary geometry data for the numerical simulation, the geometry of an existing catenary on the Gardemobanen line from the Norwegian railway network was measured. The main purpose of this paper is to find out the effect of geometrical deviations on the interaction performance of the pantograph-catenary system, namely the vertical contact quality. Therefore, only the geometrical deviations in vertical and longitudinal directions were measured. Obviously, the former directly impacts the sliding contact between the pantograph strip 
and contact wire. The latter may affect the frequency characteristics of the contact force. A laser rangefinder (Leica DISTO ${ }^{\mathrm{TM}}$ D8) was used to measure the height of critical points, including upper and lower joints of droppers, steady arm points and joints of stitch wires. The height was measured as the relative vertical height with respect to the rail. The vertical measurement is illustrated in Fig. 3, and the height $H$ of the catenary wires can be estimated by

$H=H_{\mathrm{r}}+H_{\mathrm{w}}=L_{\mathrm{r}} \cdot \sin \alpha+L_{\mathrm{w}} \cdot \sin \beta$,

where $H_{\mathrm{w}}$ is the vertical height between the critical point and the laser rangefinder, and $H_{\mathrm{r}}$ is the vertical height between the rail and the laser rangefinder; $L_{\mathrm{w}}$ and $\beta$ are respectively the distance and the corresponding pitch angle from the laser rangefinder to the contact wire; $L_{\mathrm{r}}$ and $\alpha$ are respectively the distance and the corresponding pitch angle of the rail.

The longitudinal distance $D_{1}$ of each critical point was also measured using the method illustrated in Fig. 4. To estimate the longitudinal distance $D_{1}$, the yaw angle $\gamma$ between the pole and rail is necessary. However, due to the limitation of the 6-axis inertial measurement unit (IMU) installed in the laser rangefinder, the yaw angle $\gamma$ is not convenient to measure. An alternative method is to measure the distances from the laser rangefinder to the pole $L_{\mathrm{p}}$ and to the rail $L_{\mathrm{hr}}$ and the pole-rail distance $L_{\mathrm{pr}}$. Then, the yaw angle $\gamma$ and the longitudinal distance $D_{1}$ can be estimated by

$\gamma=\arccos \left[\left(L_{\mathrm{hr}}-L_{\mathrm{pr}}\right) / L_{\mathrm{p}}\right]$,

$D_{1}=L_{\mathrm{p}} \cdot \sin \gamma=L_{\mathrm{p}} \cdot \sin \left[\arccos \left[\left(L_{\mathrm{hr}}-L_{\mathrm{pr}}\right) / L_{\mathrm{p}}\right]\right]$.

Therefore, seven parameters $L_{\mathrm{r}}, L_{\mathrm{w}}, \alpha, \beta, L_{\mathrm{p}}, L_{\mathrm{hr}}$ and $L_{\mathrm{pr}}$ need to be measured using the laser rangefinder. The accuracy of measurement in the range of $10-30 \mathrm{~m}$ can be controlled within $0.1 \mathrm{~mm}$. Moreover, the measurement

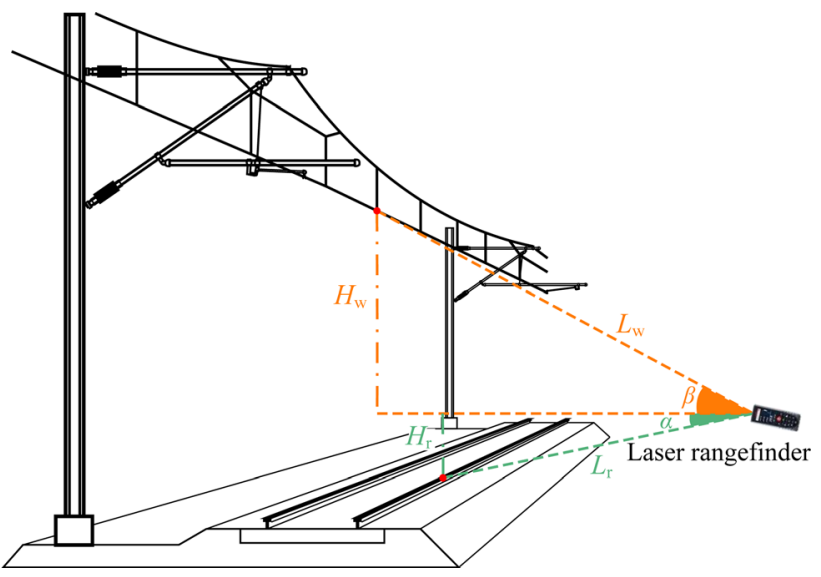

Fig. 3 The measurement of vertical geometry with the aid of a laser rangefinder

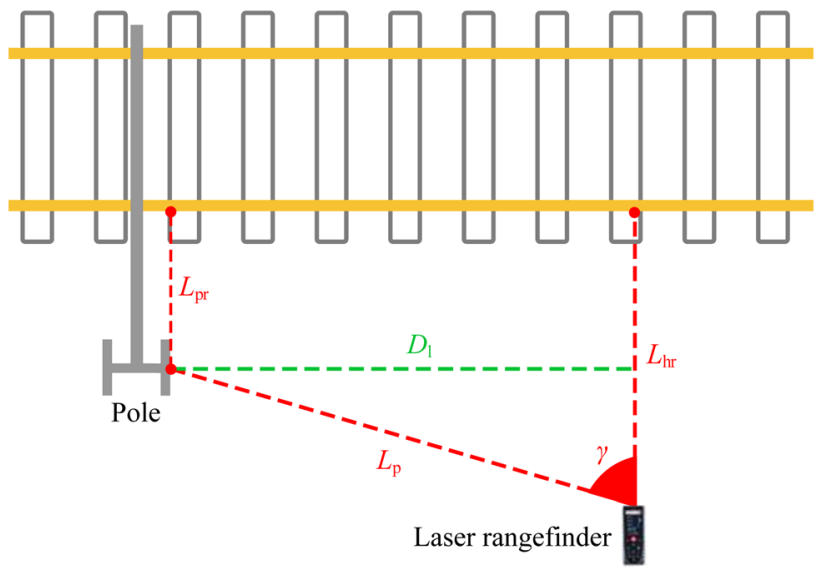

Fig. 4 The measurement of longitudinal geometry

accuracy of the pitch angles $\alpha$ and $\beta$ is controlled within $0.1^{\circ}$. Hence, the effect of any measurement errors for these two parameters on the measurement accuracy is small enough to be neglected.

Figure 5 shows the comparison of contact wire geometry within one span between the measurement and design data. The sag between two critical points is calculated based on the tension and gravity in the contact wire. It is seen that the measured geometry has a significant difference from the designed one. Especially in the vertical direction, the measured geometry shows a significant irregularity. The dropper points also show distinct deviation in the longitudinal direction. The following two approaches are utilised to describe the stochastics of deviations in each direction.

As illustrated in Fig. 6, the standard deviation of the critical point deviation against the design data is calculated for the longitudinal direction. The Gaussian distribution is employed to characterise the dispersion of longitudinal deviation, of which the probability density function (PDF) can be written as follows:

$f(x)=\frac{1}{\sigma_{\mathrm{g}} \sqrt{2 \pi}} \exp \left[-\frac{1}{2}\left(\frac{x-\mu_{\mathrm{g}}}{\sigma_{\mathrm{g}}}\right)^{2}\right]$,

where $\sigma_{\mathrm{g}}$ is the longitudinal standard deviation of critical points, which takes the value of $0.23 \mathrm{~m} ; \mu_{\mathrm{g}}$ is the corresponding mean value, which is close to zero. Following the Gaussian distribution, many samples of random longitudinal deviations are generated for all the critical points. Then, the longitudinal coordinate of each critical point can be obtained by the summation of the original position and the longitudinal deviation.

For the vertical direction, a smoothing spline is used to connect all the critical points, of which the PSD is estimated using the Yule-Walker AR method. Then, the inverse Fourier transform is implemented to generate many 


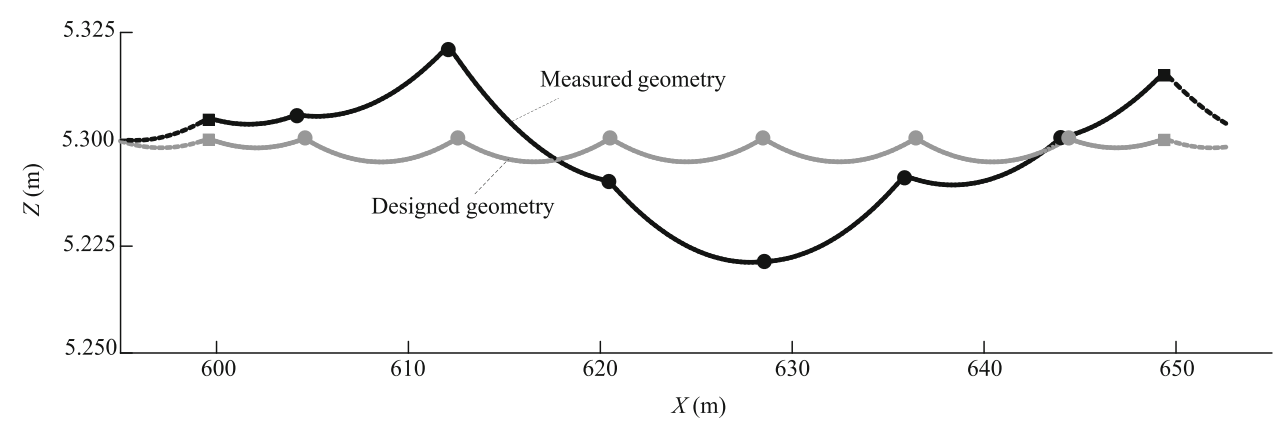

Fig. 5 Comparison of contact wire geometry between measurements and design data

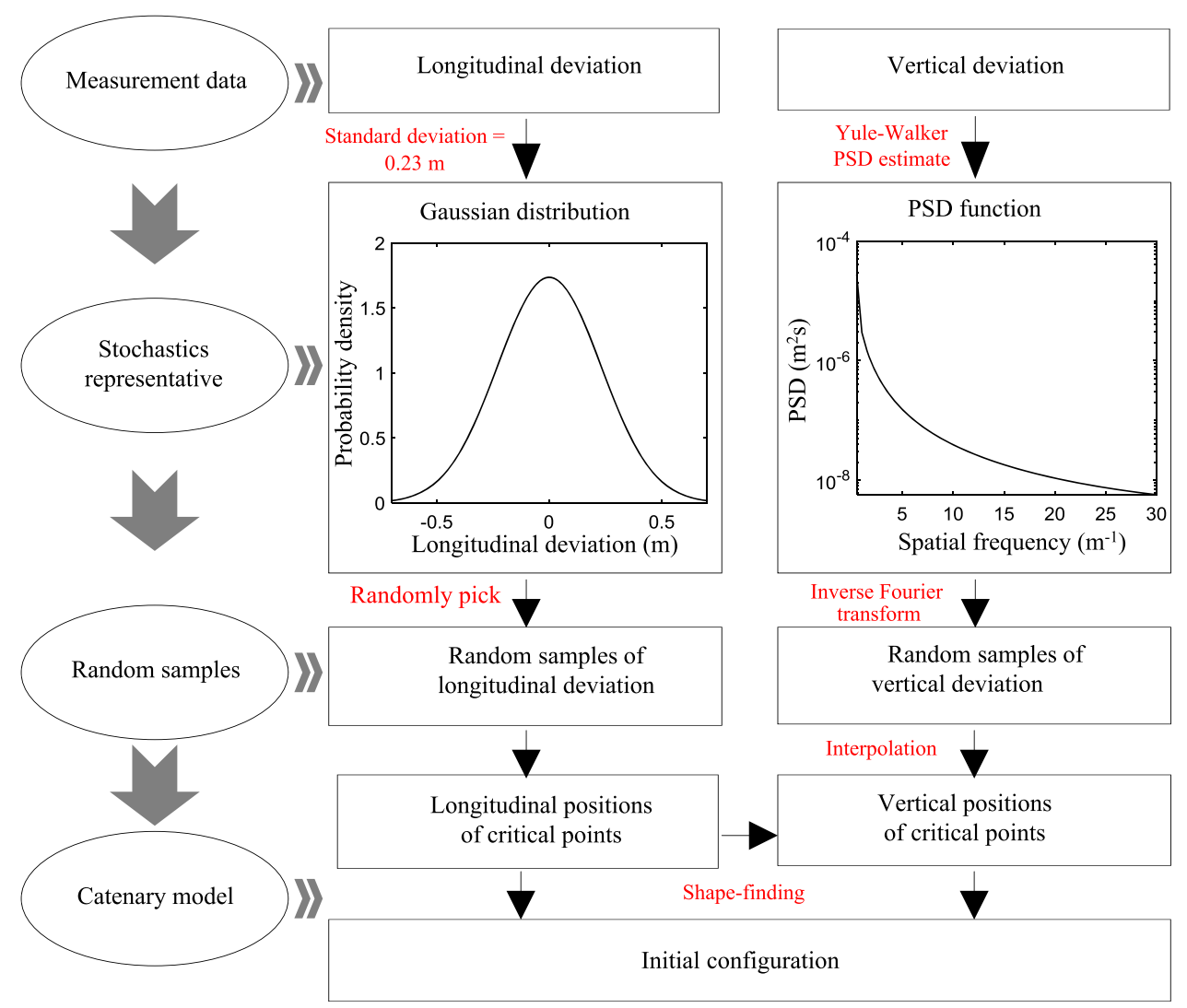

Fig. 6 Procedure to characterise stochastics of catenary geometry deviations

samples of vertical deviation from the measured PSD (Fig. 7a). The generated PSDs show good agreement with the measured one (Fig. 7b). The vertical deviations of all critical points can be obtained by the interpolation according to their longitudinal coordinates. The vertical and longitudinal coordinates of critical points are input in the catenary model to compute the initial configuration. The details of the shape-finding method are described in the next section.

\section{Modelling of pantograph-catenary system with geometry deviation}

The absolute nodal coordinate formulation (ANCF) is utilised to model the catenary in this paper, which has been proven capable of accurately describing the initial configuration [26] and addressing the geometrical nonlinearity [27]. The pantograph is represented by a developed lumped mass model with two flexible collectors to reproduce the high-frequency modes. 

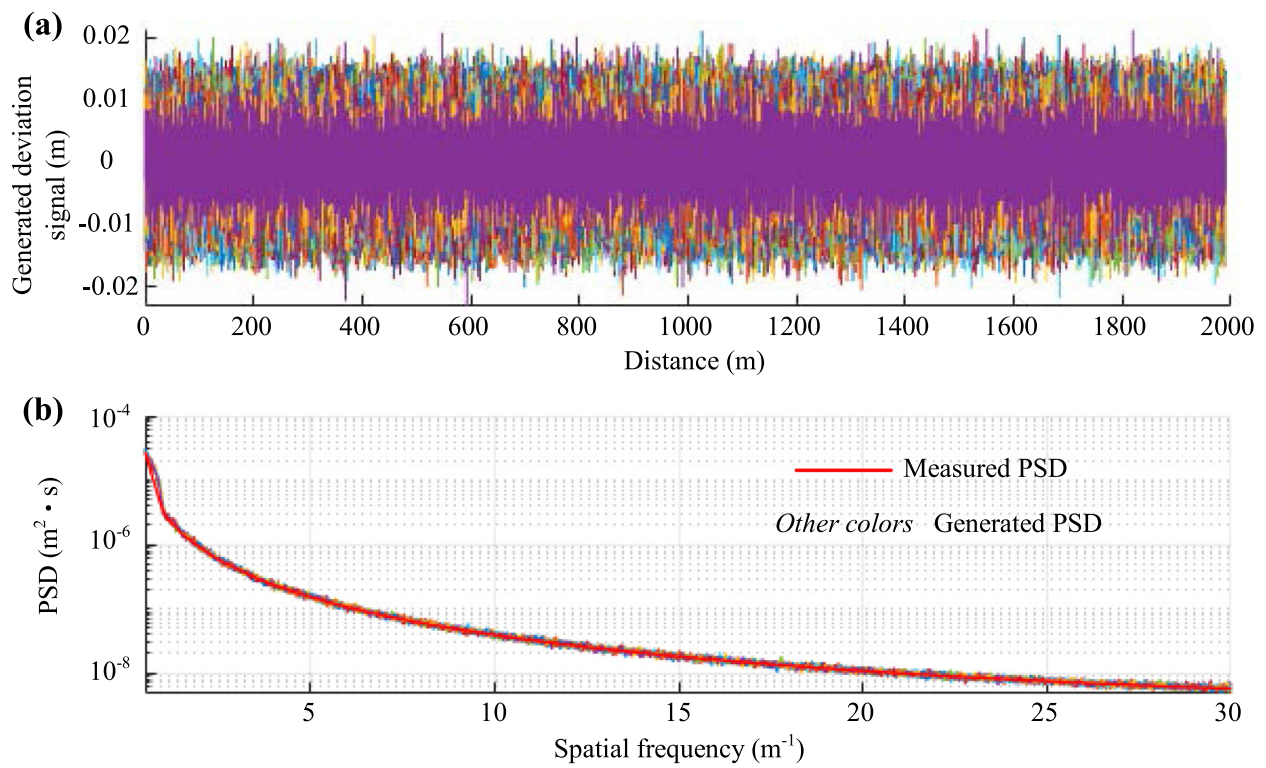

Fig. 7 a Generated deviation signal from the measured PSD; b comparison of generated and measured PSD

\subsection{ANCF beam and cable elements}

The catenary comprises a number of complex components, but its dynamic behaviour with a pantograph is normally determined by five main components: the contact wire, the messenger wire, the dropper, the stitch wire and the steady arm, as illustrated in Fig. 8. The ANCF beam element is employed here to describe the geometrical nonlinearity of messenger, contact and stitch wires. The dropper is modelled by an ANCF cable element with a nonlinear stiffness to describe different working conditions in tension and compression. The steady arm is modelled by a linear truss

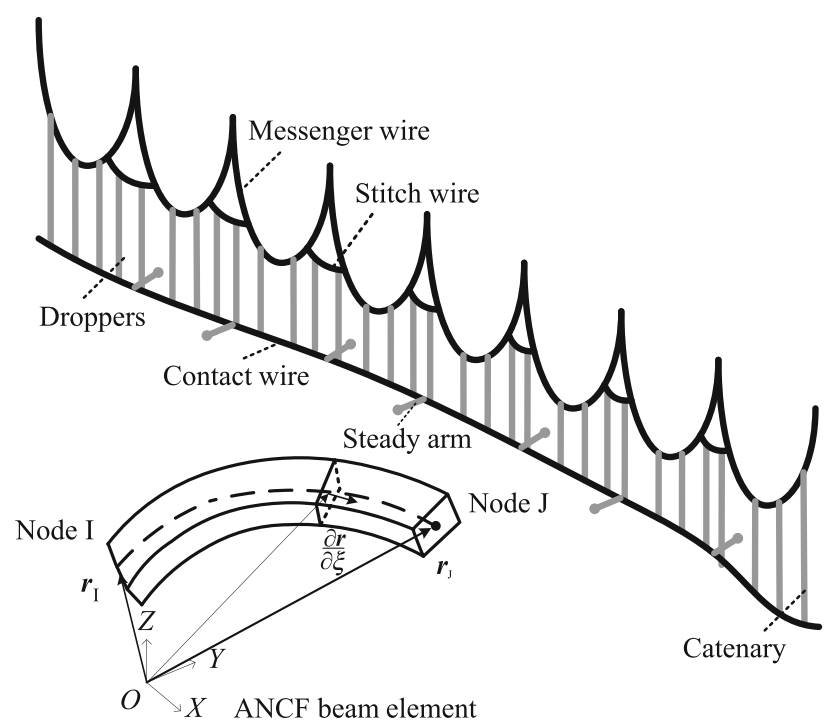

Fig. 8 The catenary model with ANCF beam and cable elements element, which can rotate around the support point. The claws on clamps are assumed as lumped masses.

In this work, the ANCF beam with 12 degrees of freedom (DOFs) is adopted to discretise the contact, messenger, and stitch wires. The elastic force vector $\boldsymbol{Q}_{\mathrm{e}}$ for each element can be expressed as the product of the secant stiffness matrix $\boldsymbol{K}_{\mathrm{e}}$ and the DOF vector $\boldsymbol{e}$ as follows:

$\boldsymbol{Q}_{\mathrm{e}}=\boldsymbol{K}_{\mathrm{e}} \boldsymbol{e}$,

where the derivation of the secant stiffness matrix $\boldsymbol{K}_{\mathrm{e}}$ can be found in [26]. In the shape-finding procedure, the tangent stiffness matrices are primarily used to calculate the incremental DOF vector $\Delta \boldsymbol{e}$ and the incremental unstrained length $\Delta L_{0}$. The corresponding tangent stiffness matrices $\boldsymbol{K}_{\mathrm{T}}$ and $\boldsymbol{K}_{\mathrm{L}}$ can be obtained by taking partial of Eq. (4) against $\boldsymbol{e}$ and $L_{0}$ as follows:

$\Delta \boldsymbol{F}=\frac{\partial \boldsymbol{Q}}{\partial \boldsymbol{e}} \Delta \boldsymbol{e}+\frac{\partial \boldsymbol{Q}}{\partial L_{0}} \Delta L_{0}=\boldsymbol{K}_{\mathrm{T}} \Delta \boldsymbol{e}+\boldsymbol{K}_{\mathrm{L}} \Delta L_{0}$,

where $\boldsymbol{F}$ is the internal force vector. A similar derivation can also be used to derive the tangent stiffness matrices of the ANCF cable element. It should be noted that when the dropper works in slackness, the axial stiffness changes to zero. Assembling the stiffness matrix of each element yields the global incremental equilibrium equation for the whole catenary as follows:

$\Delta \boldsymbol{F}^{\mathrm{G}}=\boldsymbol{K}_{\mathrm{T}}^{\mathrm{G}} \Delta \boldsymbol{U}_{\mathrm{C}}+\boldsymbol{K}_{\mathrm{L}}^{\mathrm{G}} \Delta \boldsymbol{L}_{0}$,

where $\Delta \boldsymbol{F}^{\mathrm{G}}$ is the global unbalanced force vector. $\boldsymbol{K}_{\mathrm{T}}^{\mathrm{G}}$ and $\boldsymbol{K}_{\mathrm{L}}^{\mathrm{G}}$ are the global stiffness matrices related to the incremental nodal displacement vector $\Delta \boldsymbol{U}_{\mathrm{C}}$ and the incremental unstrained length vector $\Delta \boldsymbol{L}_{0}$, respectively. The initial 
configuration of the catenary can be calculated by solving Eq. (6). The details of the initialisation procedure are discussed in the following section.

\subsection{Computation of initial configuration with contact line height variability}

It can be seen that Eq. (6) cannot be solved directly because $\left[\begin{array}{ll}\boldsymbol{K}_{\mathrm{T}}^{\mathrm{G}} & \boldsymbol{K}_{\mathrm{L}}^{\mathrm{G}}\end{array}\right]$ is not a square matrix. The total number of unknowns exceeds the total number of equations, which leads to undetermined solutions. Therefore, additional constraint conditions should be provided to reduce the number of unknowns and ensures that Eq. (6) has unique solutions. These additional constraint conditions are determined by the design specification and the measurement geometry data. In this work, three types of additional constraints are defined, illustrated in Fig. 9.

- The vertical positions of all dropper points in the contact wire are restricted according to the geometry data.

- The longitudinal direction of each node is restricted to suppress the longitudinal movement.

- The designing tensions are imposed on the endpoints of messenger, contact and stitch lines.

These constraint conditions reduce $\left[\begin{array}{ll}\boldsymbol{K}_{\mathrm{T}}^{\mathrm{G}} & \boldsymbol{K}_{\mathrm{L}}^{\mathrm{G}}\end{array}\right]$ in Eq. (6) to be a square matrix, which ensures the equality between the numbers of equations and unknowns. The steady arm points are fixed tentatively in the iterative procedure to place the steady arm points at their correct positions. The inclination of each steady arm can be calculated by the resistance forces in the fixed point, which is replaced by a rigid truss element without rotation DOFs to reproduce the realistic behaviour of a steady arm. It should be noted that these additional constraints are only used in the shape-finding procedure. They are removed after the equilibrium state is achieved.

An example is presented here using the parameters of the measured catenary in Sect. 2. The main property parameters of the catenary are collected in Table 1. Firstly, each dropper's longitudinal position and steady arm point are modified by the random longitudinal deviation generated from $N\left(u_{\mathrm{g}}, \delta_{\mathrm{g}}^{2}\right)$. Using one sample of vertical deviation in Fig. 8a, the dropper's heights and steady arm point can be extracted by interpolation according to their longitudinal positions. The convergence condition is defined as follows:

$\max \left(\left|\left[\begin{array}{c}\Delta \boldsymbol{U}_{\mathrm{C}} \\ \Delta \boldsymbol{L}_{0}\end{array}\right]\right|\right)<10^{-9} \mathrm{~m}$.

The maximum absolute residual versus the cycle index is presented in Fig. 10. It is seen that the convergence condition can be satisfied after 18 cycles of iteration. The results of catenary geometry are presented in Fig. 11. It is seen that the deviated configuration has a distinct difference from the original one. In the next section, a pantograph model is included to investigate the contact force's dispersion with the effect of geometry deviation. The central nine spans are chosen as the contact force analysis range to eliminate the boundary effect interference.

Table 1 Catenary property parameters

\begin{tabular}{ll}
\hline Parameter & Value \\
\hline Total length $(\mathrm{km})$ & 1.012 \\
Contact wire tension $(\mathrm{kN})$ & 15 \\
Messenger wire tension $(\mathrm{kN})$ & 15 \\
Stitch wire tension $(\mathrm{kN})$ & 2.8 \\
Contact wire area $\left(\mathrm{mm}^{2}\right)$ & 120 \\
Messenger wire area $\left(\mathrm{mm}^{2}\right)$ & 65.8 \\
Stitch wire area $\left(\mathrm{mm}^{2}\right)$ & 3.44 \\
Contact wire linear density $(\mathrm{kg} / \mathrm{m})$ & 1.07 \\
Messenger wire linear density $(\mathrm{kg} / \mathrm{m})$ & 0.596 \\
Number of spans in contact with the pantograph & 18 \\
\hline
\end{tabular}

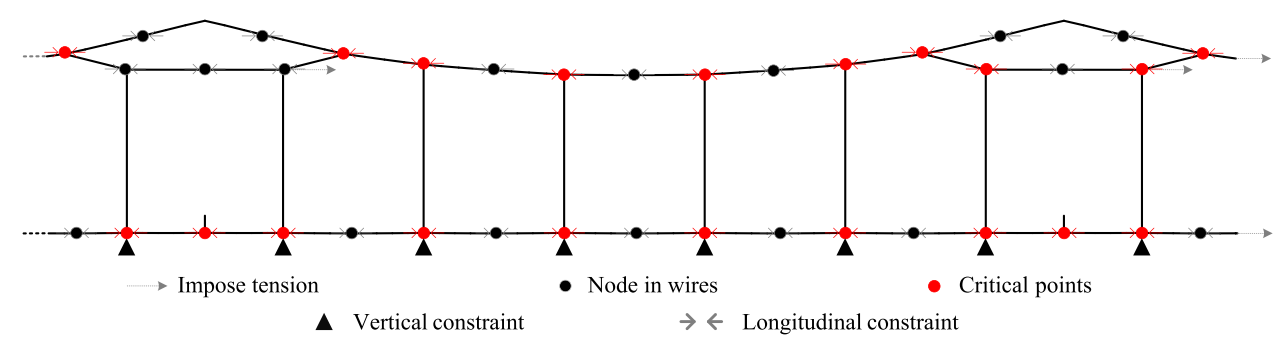

Fig. 9 Illustration of additional constraints in the shape-finding of catenary 


\subsection{Pantographs-catenary interaction formulation}

To evaluate the response at high frequencies, the pantograph is assumed to be a lumped mass model with flexible collectors, as shown in Fig. 12. Each collector is discretised into a few 2D beam elements. The stiffness matrix can be written as follows:

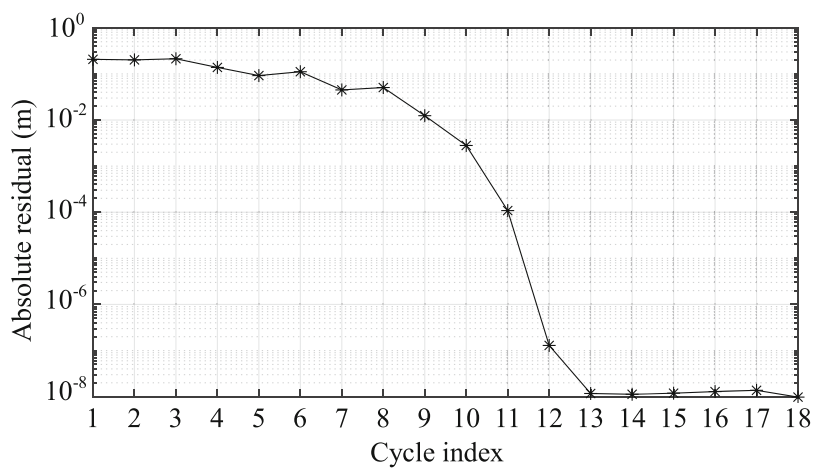

Fig. 10 Absolute residual in shape-finding procedure
$\boldsymbol{k}_{\mathrm{pe}}=\frac{E I_{\mathrm{pe}}}{l_{\mathrm{pe}}^{3}}\left[\begin{array}{cccc}12 & 6 l_{\mathrm{pe}} & -12 & 6 l_{\mathrm{pe}} \\ 6 l_{\mathrm{pe}} & 4 l_{\mathrm{pe}}^{2} & -6 l_{\mathrm{pe}} & 2 l_{\mathrm{pe}}^{2} \\ -12 & -6 l_{\mathrm{pe}} & 12 & -6 l_{\mathrm{pe}} \\ 6 l_{\mathrm{pe}} & 2 l_{\mathrm{pe}}^{2} & -6 l_{\mathrm{pe}} & 4 l_{\mathrm{pe}}^{2}\end{array}\right]$,

where $l_{\mathrm{pe}}$ is the element length and $E I_{\mathrm{pe}}$ is the bending stiffness of the collector. Usually, the collector is made of two types of materials, namely graphite and aluminium. The bending stiffness of the collector is calculated by the mixture theory [28]. As the flexibility of the pantograph framework is very low [29], it is treated as a rigid lumped mass here. A penalty function method is employed to couple the contact wire and the collector. Therefore, the contact force $f_{\mathrm{c}}$ can be calculated by

$f_{\mathrm{c}}=\left\{\begin{array}{lll}k_{\mathrm{s}}\left(z_{\mathrm{p}}-z_{\mathrm{c}}\right) & \text { if } & z_{\mathrm{p}}-z_{\mathrm{c}}>0 \\ 0 & \text { if } & z_{\mathrm{p}}-z_{\mathrm{c}} \leq 0\end{array}\right.$,

in which $z_{\mathrm{p}}$ is the pantograph head uplift, and $z_{\mathrm{c}}$ is the vertical displacement of the contact wire in the contact
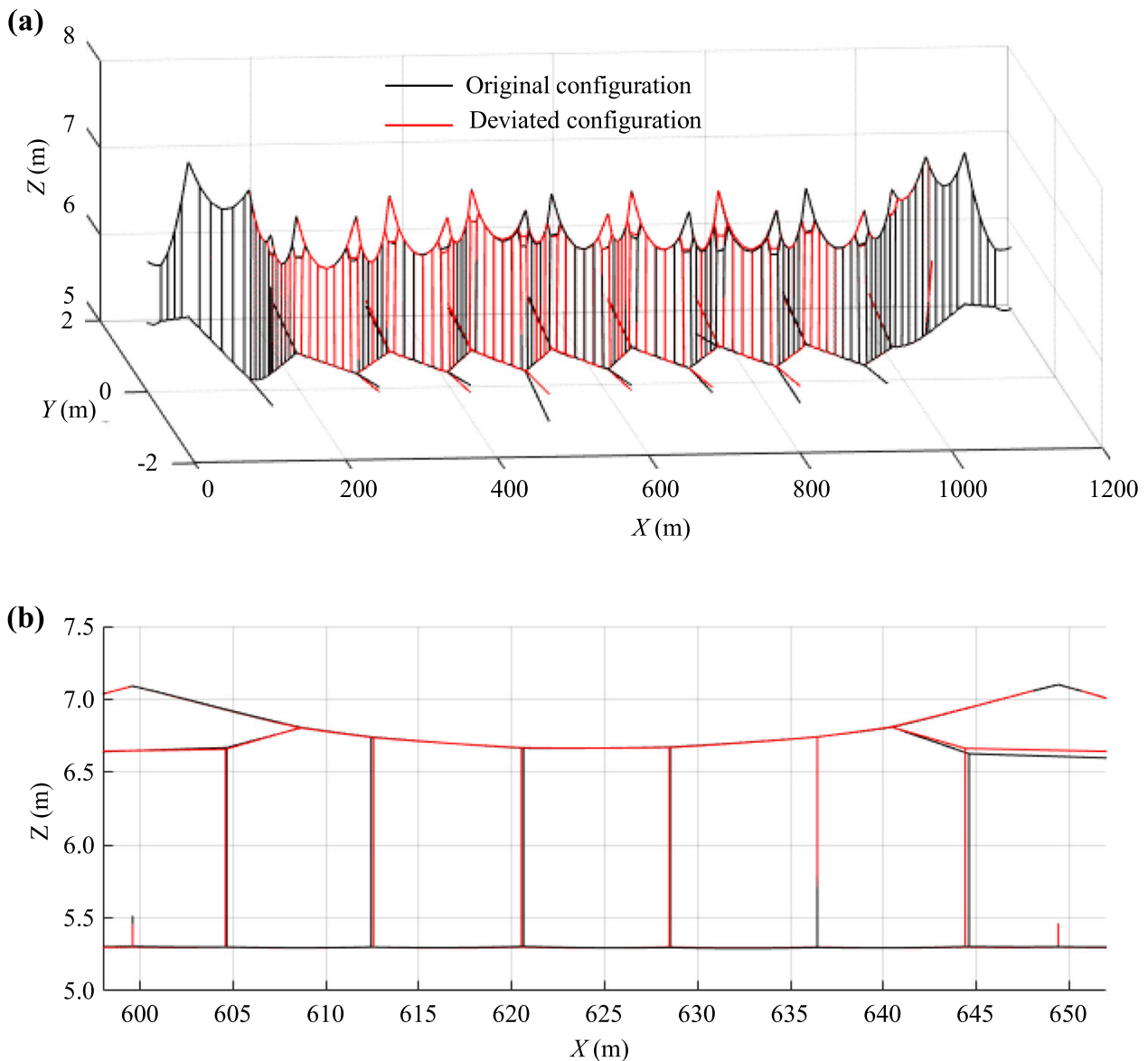

Fig. 11 Results of catenary geometry: a full geometry; b locally enlarged view of one span 


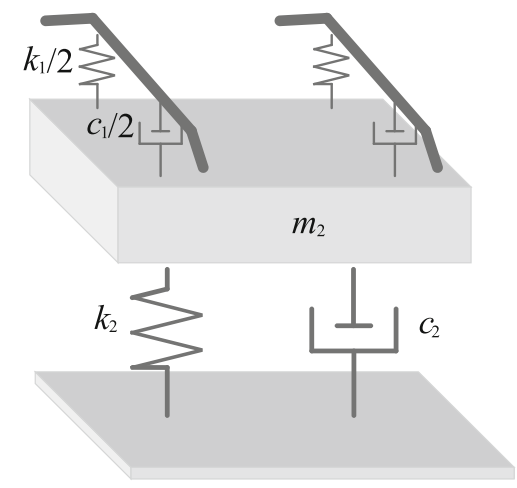

Fig. 12 Lumped mass model of pantograph with flexible collectors

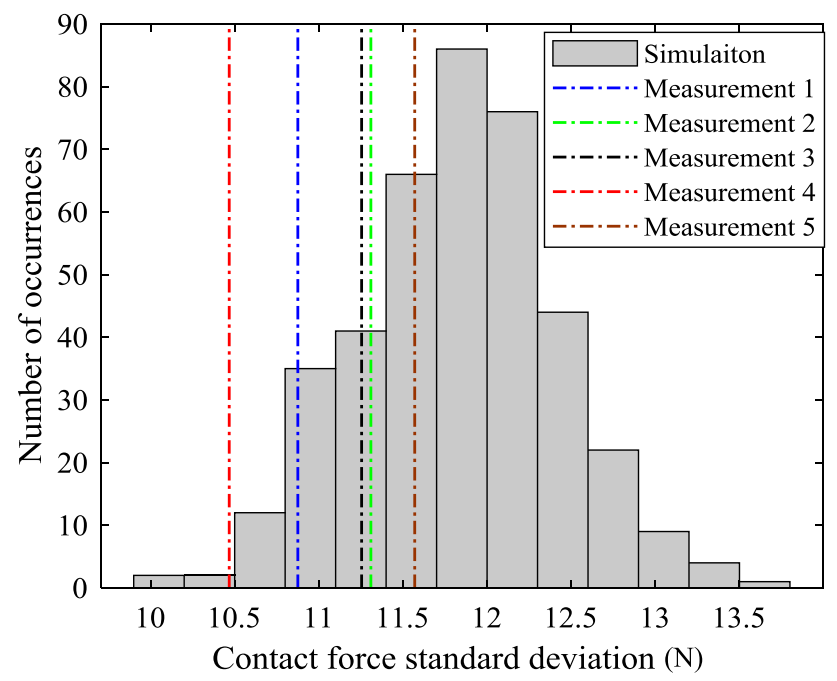

Fig. 13 Histogram of simulated contact force standard deviation. Dash lines denote the measured contact force standard deviations from five times of field tests

point. Using Eq. (9), the equation of motion for the pantograph-catenary system is written as

$\boldsymbol{M}_{\mathrm{G}} \ddot{\boldsymbol{U}}(t)+\boldsymbol{C}_{\mathrm{G}} \dot{\boldsymbol{U}}(t)+\boldsymbol{K}_{\mathrm{G}}(t) \boldsymbol{U}(t)=\boldsymbol{F}_{\mathrm{G}}(t)$,

where $\boldsymbol{M}_{\mathrm{G}}, \boldsymbol{C}_{\mathrm{G}}$ and $\boldsymbol{K}_{\mathrm{G}}(t)$ are the mass, damping and stiffness matrices for the whole system, respectively; $\boldsymbol{F}_{\mathrm{G}}(t)$ is the external force vector. A Newmark integration scheme is adopted to solve Eq. (10). The stiffness matrix $\boldsymbol{K}_{\mathrm{G}}(t)$ is updated according to the catenary deformation in each time step to describe the geometrical nonlinearity and dropper slackness [15].

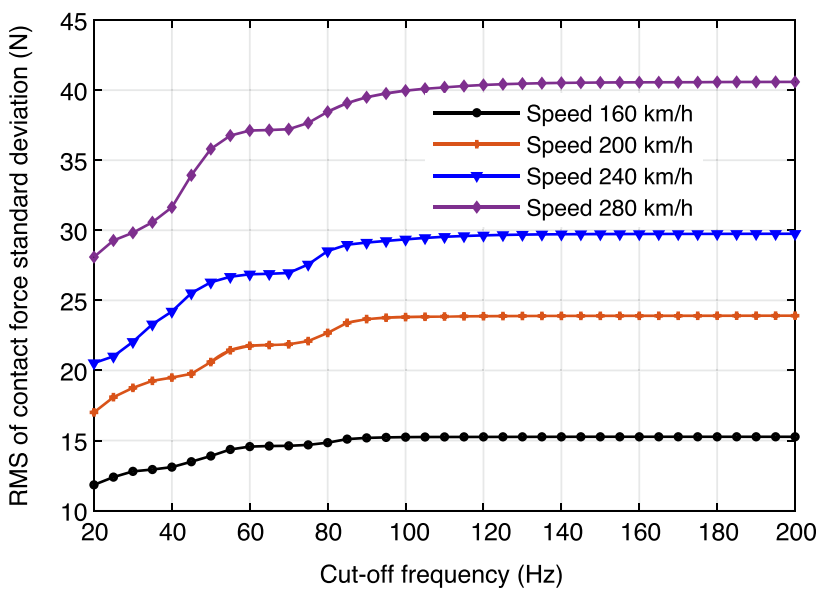

Fig. 14 RMS of contact force standard deviation with different cutoff frequencies

\section{Effect of geometry deviation on contact forces}

This section investigates the effect of catenary geometry deviation on the contact force through a series of numerical simulations. The measured contact forces collected by an inspection vehicle regularly running in the Norwegian network are compared with the simulation results. Then, the effect of the cut-off frequency on the simulation results is investigated. Finally, probabilistic analyses are performed on the simulated data to assess the interaction performance of the pantograph-catenary system. In Sect. 4.1, the train speed is set as $160 \mathrm{~km} / \mathrm{h}$, consistent with the inspection vehicle's speed. In Sects. 4.2 and 4.3, the train speed changes from 160 to $280 \mathrm{~km} / \mathrm{h}$ to investigate the dynamic performance at different speeds. It should be noted that $280 \mathrm{~km} / \mathrm{h}$ is the catenary top speed, which is defined as 0.7 times the wave propagation speed according to En 50119 [30].

\subsection{Comparison with measurement data}

Based on the idea of the Monte Carlos method, 400 numerical simulations are performed to describe the dispersion of dynamic responses. According to En 50367 [31], the contact force standard deviation filtered within $0-20 \mathrm{~Hz}$ is the most critical assessment indicator to describe the comprehensive current collection quality. Therefore, the histogram of the contact force standard deviation obtained by 400 simulations is presented in Fig. 13. The standard deviation of the simulated contact force generally follows a symmetric distribution with a standard deviation of $0.59 \mathrm{~N}$ and a mean of $11.82 \mathrm{~N}$. The measured contact force standard deviations from five independent field tests are denoted by dash lines in Fig. 13. The measured contact forces are obtained by the inspection 

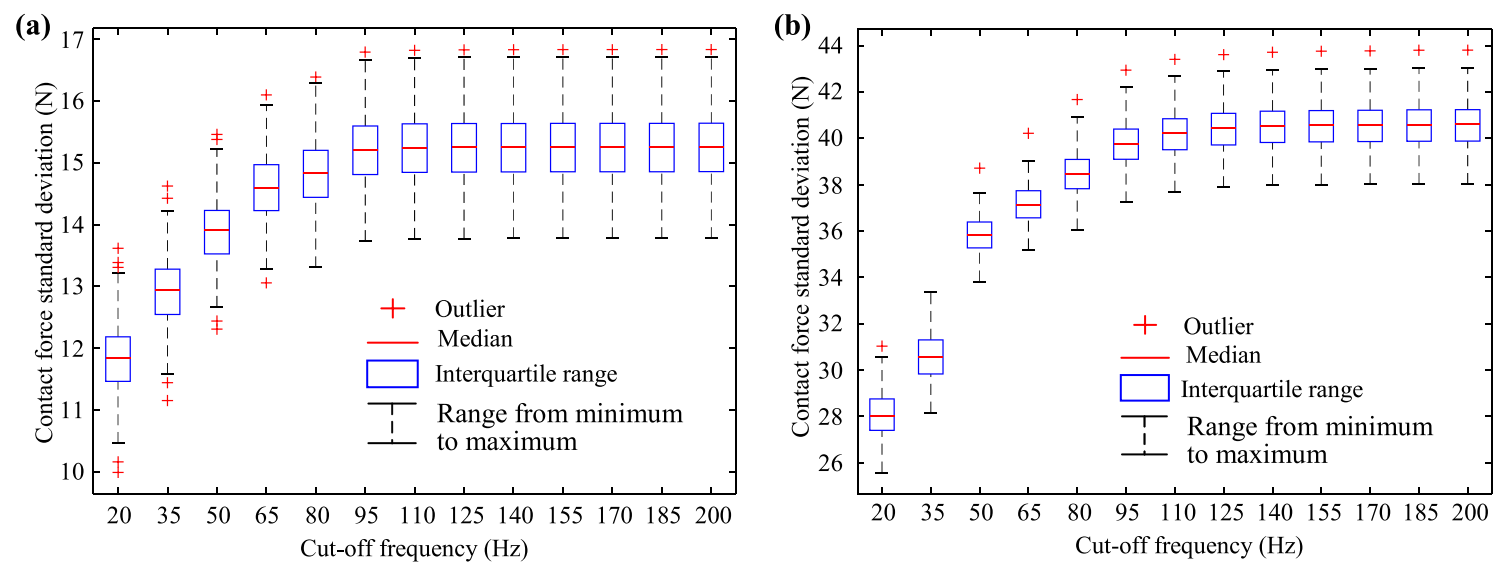

Fig. 15 Boxplots of contact force standard deviation with different cut-off frequencies at a $160 \mathrm{~km} / \mathrm{h}$ and b $280 \mathrm{~km} / \mathrm{h}$
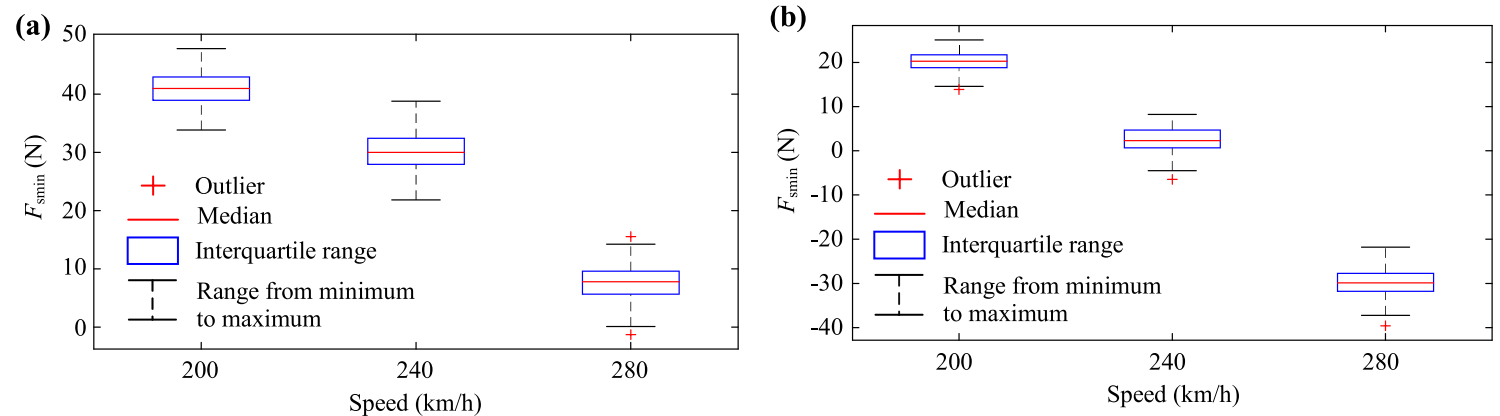

Fig. 16 Boxplots of statistical minimum contact force at different operating speeds with cut-off frequencies of a $20 \mathrm{~Hz}$ and b $200 \mathrm{~Hz}$

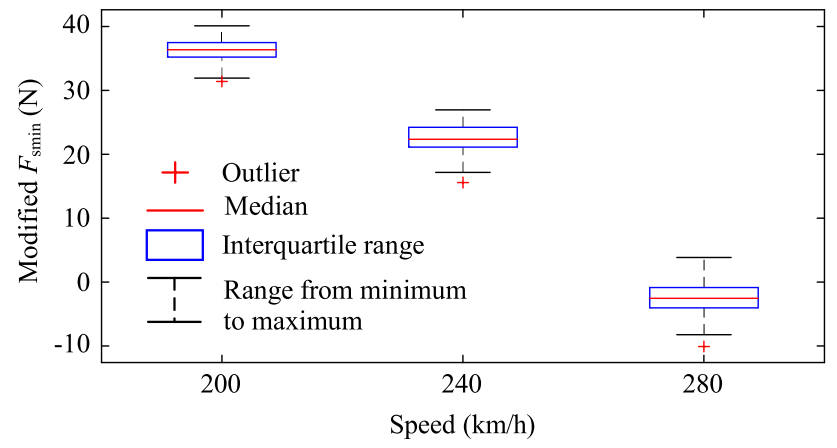

Fig. 17 Boxplots of modified statistical minimum contact force at different operating speeds with $200 \mathrm{~Hz}$ cut-off frequency

vehicle regularly running in the Norwegian network. The average time interval between two adjacent measurements is six months. All these measured contact force standard deviations are within the scope of the simulation results. However, all the measurement results are in the 'left part' of the distribution, which can be explained from two aspects. The first one is that the bad performance in the right part is not likely to happen in the field test due to the appropriate maintenance of the railway operator. The second explanation is that the average of the simulation results cannot accurately represent the actual condition, and the simulation results are expected to be calibrated according to the measurement data. However, both explanations cannot be fully verified at present because only a very limited amount of measurement data are available. The actual distribution of contact force statistics deserves to be revealed with abundant measurement data in the future.

\subsection{Analysis with different cut-off frequencies}

The current standard En 50367 specifies a frequency range of interest within $0-20 \mathrm{~Hz}$. This low-frequency range is expected to be improved to capture more high-frequency behaviour of the pantograph-catenary interaction. It should be noted that the high-frequency measurement data cannot be provided to validate the simulation results, which is beyond the frequency range of the current measurement equipment. The work in this section only conducts a qualitative analysis to point out the expected impact of the geometry deviation on the contact force when the cut-off frequency moves up to $200 \mathrm{~Hz}$. In this numerical simulation, a small element size of the contact wire and a pantograph model with flexible collectors are adopted to ensure numerical accuracy at high frequencies. The train 


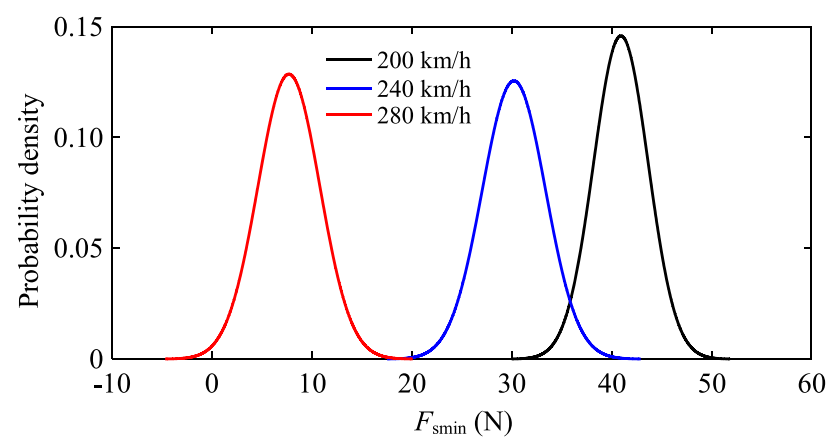

Fig. 18 Probability density of statistical minimum contact force at different operating speeds with $20 \mathrm{~Hz}$ cut-off frequency

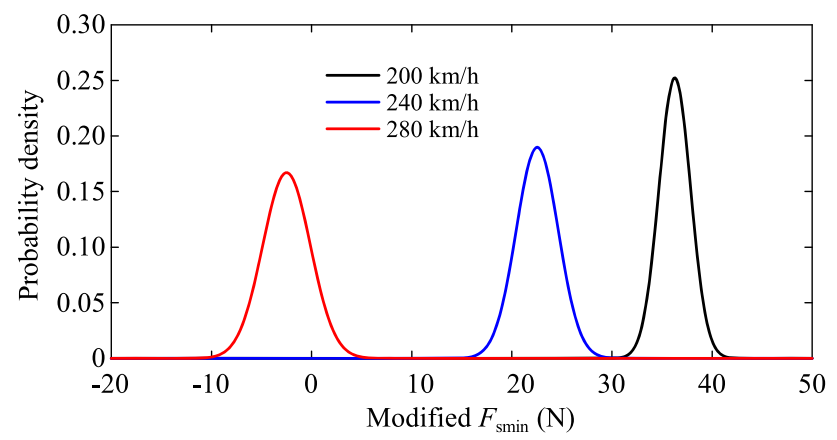

Fig. 19 Probability density of modified statistical minimum contact force at different operating speeds with $200 \mathrm{~Hz}$ cut-off frequency

speed is chosen from 160 to $280 \mathrm{~km} / \mathrm{h}$ with an interval of $40 \mathrm{~km} / \mathrm{h}$. For each speed, 400 simulations are performed to ensure that the dispersion can be reflected. Figure 14 shows the root mean square (RMS) of the contact force standard deviation with different cut-off frequencies. It is seen that the RMS undergoes a significant increase with the cut-off frequency increasing and then becomes stable after the cutoff frequency reaches a critical value. Generally, the critical value increases with the improvement of the speed. At $160 \mathrm{~km} / \mathrm{h}$, the RMS becomes stable after the cut-off frequency is larger than $90 \mathrm{~Hz}$. The critical cut-off frequencies are about 105, 125 and $150 \mathrm{~Hz}$ at the speed of 200, 240 and $280 \mathrm{~km} / \mathrm{h}$, respectively. The corresponding spatial wavelength is around $0.5 \mathrm{~m}$, about $1 / 16$ of the interval between two adjacent droppers. According to the analysis in [26], the cut-off frequency should cover the $1 / 4$ of dropper interval to fully describe the catenary's geometry effect in the contact force. However, the geometry deviation is not considered in [26]. Thus, this critical cut-off frequency should be improved to cover the $1 / 16$ of the dropper interval to describe the effect of the catenary geometry deviation fully. The boxplots of contact force standard deviation with different cut-off frequencies at 160 and $280 \mathrm{~km} / \mathrm{h}$ are presented in Fig. 15. It is seen that the fluctuating range of the contact force standard deviation does not show a significant change when the cut-off frequency increases over the critical value.

\subsection{Probability analysis}

In assessing current collection quality, the statistical minimum contact force is a critical index and should be positive to restrict the occurrence of contact loss, which is calculated by

$F_{\text {smin }}=F_{\text {mean }}-3 \delta$,

where $F_{\text {mean }}$ is the mean contact force and $\delta$ is the contact force standard deviation. The coefficient ' 3 ' is determined by the $99.73 \%$ confidence level. In this section, the statistical minimum contact force at each speed is calculated to check the interaction performance with random geometry deviation of catenary. Figure 16a, b presents the boxplots of statistical minimum contact force at different operating speeds with the cut-off frequencies of 20 and $200 \mathrm{~Hz}$, respectively. At $200 \mathrm{~km} / \mathrm{h}$, the statistical minimum contact forces are always positive with 20 and $200 \mathrm{~Hz}$ cut-off frequencies, which satisfies the assessment standard. Actually, $200 \mathrm{~km} / \mathrm{h}$ is the operating speed for the analysed catenary system. When the speed moves up to $240 \mathrm{~km} / \mathrm{h}$, some negative statistical minimum contact forces can be observed with the $200 \mathrm{~Hz}$ cut-off frequency. At $280 \mathrm{~km} / \mathrm{h}$, the negative statistical minimum contact force can be observed with the $20 \mathrm{~Hz}$ cut-off frequency. Almost all statistical minimum contact forces are smaller than $0 \mathrm{~N}$ with $200 \mathrm{~Hz}$ cut-off frequency at $280 \mathrm{~km} / \mathrm{h}$. According to En 50119 [30], $280 \mathrm{~km} / \mathrm{h}$ is very close to the catenary's top speed, which is defined as 0.7 times the wave propagation speed. When the train speed is close to the top speed, unsatisfactory current collection quality can be expected. But it should be noted that not all the contact loss at $200 \mathrm{~Hz}$ cut-off frequency is unacceptable. According to the experience of railway operators, a certain amount of contact loss is tolerant at high frequency. Thus, the definition of the statistical minimum contact force should be modified. Assuming that $1 \%$ contact loss is acceptable at $200 \mathrm{~Hz}$, the modified statistical minimum contact force can be calculated by

$F_{\text {smin }}=F_{\text {mean }}-2.326 \delta$,

where the coefficient ' 2.326 ' is determined by the $98 \%$ confidence level, which means that $1 \%$ negative contact force can be tolerant. The boxplots of modified statistical minimum contact force at different operating speeds with $200 \mathrm{~Hz}$ cut-off frequency are presented in Fig. 17. It is seen that all the modified statistical minimum contact forces are above zero at speeds of 200 and $240 \mathrm{~km} / \mathrm{h}$. At $280 \mathrm{~km} / \mathrm{h}$, most results are no longer acceptable. 
Figure 18 presents the probability densities of statistical minimum contact force at different operating speeds with the cut-off frequencies of $20 \mathrm{~Hz}$. The statistical minimum contact forces with $20 \mathrm{~Hz}$ cut-off frequency are always larger than the threshold $0 \mathrm{~N}$ at 200 and $240 \mathrm{~km} / \mathrm{h}$. At $280 \mathrm{~km} / \mathrm{h}$, the statistical minimum contact force has a $0.68 \%$ possibility to be negative. When the cut-off frequency moves up to $200 \mathrm{~Hz}$, the probability densities of modified statistical minimum contact force at different operating speeds are presented in Fig. 19. It is seen that the modified statistical minimum contact force has an $85.58 \%$ possibility to be negative at $280 \mathrm{~km} / \mathrm{h}$. If fewer contact losses are preferred, the pantograph has to be uplifted to have a higher mean contact force. It should be noted that the threshold of $1 \%$ for the contact loss is just an example here to demonstrate the probability analysis. The railway operator should give a more reasonable value according to actual conditions.

\section{Conclusions}

This paper presents a field measurement of the catenary geometry from the Norwegian railway network, providing realistic catenary geometry data for the numerical simulation. Based on the Monte Carlo method, many contact wire geometry samples are generated and included in the catenary model. Employing a pantograph model with flexible collectors, the effect of random geometry deviation on the contact force is investigated. The main conclusions are drawn as follows:

(1) The catenary geometry deviation causes a significant dispersion of the response of pantograph-catenary interaction. The measured contact forces by the inspection vehicle from the field tests are within the scope of the simulation results.

(2) The critical cut-off frequency should be improved to cover $1 / 16$ of the dropper interval, which can fully describe the effect of the catenary's geometry deviation on the contact force.

(3) The statistical minimum contact force can be modified according to the tolerant contact loss rate at high frequency. The probability analysis indicates that modified statistical minimum contact force has an $85.58 \%$ possibility to be negative at $280 \mathrm{~km} / \mathrm{h}$. The pantograph-catenary system has an unpleasant interaction performance at the catenary top speed when the random catenary geometry deviation is included.

Acknowledgements This work is funded by the Norwegian Railway Directorate.
Open Access This article is licensed under a Creative Commons Attribution 4.0 International License, which permits use, sharing, adaptation, distribution and reproduction in any medium or format, as long as you give appropriate credit to the original author(s) and the source, provide a link to the Creative Commons licence, and indicate if changes were made. The images or other third party material in this article are included in the article's Creative Commons licence, unless indicated otherwise in a credit line to the material. If material is not included in the article's Creative Commons licence and your intended use is not permitted by statutory regulation or exceeds the permitted use, you will need to obtain permission directly from the copyright holder. To view a copy of this licence, visit http://creativecommons. org/licenses/by/4.0/.

\section{References}

1. Cho YH, Lee K, Park Y et al (2010) Influence of contact wire pre-sag on the dynamics of pantograph railway catenary. Int $\mathrm{J}$ Mech Sci 52:1471-1490

2. Song Y, Antunes P, Pombo J, Liu Z (2020) A methodology to study high-speed pantograph-catenary interaction with realistic contact wire irregularities. Mech Mach Theory 152:103940

3. Song Y, Liu Z, Ronnquist A et al (2020) Contact wire irregularity stochastics and effect on high-speed railway pantograph-catenary interactions. IEEE Trans Instrum Meas 69:8196-8206

4. Nåvik P, Rønnquist A, Stichel S (2017) Variation in predicting pantograph-catenary interaction contact forces, numerical simulations and field measurements. Veh Syst Dyn 55:1265-1282

5. Zhai W (2020) Vehicle-track coupled dynamics: theory and application. Springer, Singapore

6. Bruni S, Bucca G, Carnevale M et al (2018) Pantograph-catenary interaction: recent achievements and future research challenges. Int J Rail Transp 6:57-82

7. Zhang W, Zou D, Tan M et al (2018) Review of pantograph and catenary interaction. Front Mech Eng 13:311-322

8. Wu TX, Brennan MJ (1999) Dynamic stiffness of a railway overhead wire system and its effect on pantograph-catenary system dynamics. J Sound Vib 219:483-5029

9. VoVan O, Massat JP, Balmes E (2017) Waves, modes and properties with a major impact on dynamic pantograph-catenary interaction. J Sound Vib 402:51-69

10. Song Y, Rønnquist A, Jiang T, Nåvik P (2021) Identification of short-wavelength contact wire irregularities in electrified railway pantograph-catenary system. Mech Mach Theory 162:104338

11. Jiang T, Frøseth GT, Rønnquist A, Fagerholt E (2020) A robust line-tracking photogrammetry method for uplift measurements of railway catenary systems in noisy backgrounds. Mech Syst Signal Process 144:106888

12. Xu L, Zhai W (2020) Train-track coupled dynamics analysis: system spatial variation on geometry, physics and mechanics. Railw Eng Sci 28:36-53

13. Song Y, Wang Z, Liu Z, Wang R (2021) A spatial coupling model to study dynamic performance of pantograph-catenary with vehicle-track excitation. Mech Syst Signal Process 151:107336

14. Song Y, Zhang M, Wang H (2021) A response spectrum analysis of wind deflection in railway overhead contact lines using pseudo-excitation method. IEEE Trans Veh Technol 70:1169-1178

15. Song Y, Liu Z, Xu Z, Zhang J (2019) Developed moving mesh method for high-speed railway pantograph-catenary interaction based on nonlinear finite element procedure. Int J Rail Transp $7: 173-190$ 
16. Kobayashi S, Stoten DP, Yamashita Y, Usuda T (2019) Dynamically substructured testing of railway pantograph/catenary systems. Proc Inst Mech Eng Part F J Rail Rapid Transit 233:516-525

17. Gregori S, Tur M, Nadal E et al (2017) Fast simulation of the pantograph-catenary dynamic interaction. Finite Elem Anal Des 129:1-13

18. Zhu S, Luo J, Wang M, Cai C (2020) Mechanical characteristic variation of ballastless track in high-speed railway: effect of train-track interaction and environment loads. Railw Eng Sci 28:408-423

19. Vesali F, Rezvani MA, Molatefi H, Hecht M (2019) Static formfinding of normal and defective catenaries based on the analytical exact solution of the tensile Euler-Bernoulli beam. Proc Inst Mech Eng Part F J Rail Rapid Transit 233:691-700

20. Song Y, Liu Z, Lu X (2020) Dynamic performance of high-speed railway overhead contact line interacting with pantograph considering local dropper defect. IEEE Trans Veh Technol 69:5958-5967

21. Zhang W, Mei G, Zeng J (2003) A study of pantograph/catenary system dynamics with influence of presag and irregularity of contact wire. Veh Syst Dyn 37:593-604

22. Song Y, Wang H, Liu Z (2021) An investigation on the current collection quality of railway pantograph-catenary systems with contact wire wear degradations. IEEE Trans Instrum Meas 70:1-11

23. Nåvik P, Rønnquist A, Stichel S (2016) The use of dynamic response to evaluate and improve the optimization of existing soft railway catenary systems for higher speeds. Proc Inst Mech Eng Part F J Rail Rapid Transit 230:1388-1396
24. Van OV, Massat JP, Laurent C, Balmes E (2014) Introduction of variability into pantograph-catenary dynamic simulations. Veh Syst Dyn 52:1254-1269

25. Gregori S, Tur M, Tarancón JE, Fuenmayor FJ (2019) Stochastic Monte Carlo simulations of the pantograph-catenary dynamic interaction to allow for uncertainties introduced during catenary installation. Veh Syst Dyn 57:471-492

26. Song Y, Rønnquist A, Nåvik P (2020) Assessment of the highfrequency response in railway pantograph-catenary interaction based on numerical simulation. IEEE Trans Veh Technol 69:10596-10605

27. Kulkarni S, Pappalardo CM, Shabana AA (2017) Pantograph/catenary contact formulations. J Vib Acoust Trans ASME 139:011010

28. Soboyejo W (2002) Mechanical properties of engineered materials. CRC Press, New York

29. Nåvik P, Derosa S, Rønnquist A (2021) On the use of experimental modal analysis for system identification of a railway pantograph. Int J Rail Transp 9:132-143

30. European Committee for Electrotechnical Standardization (2013) EN 50119:2009+A1:2013 Railway applications-fixed installations-electric traction overhead contact lines. European Standards (EN), Brussels

31. European Committee for Electrotechnical Standardization (2016) EN 50367 Railway applications-current collection systemstechnical criteria for the interaction between pantograph and overhead line. European Standards (EN), Brussels 